\begin{tabular}{|c|c|}
\hline Title & Direct interaction between EFL1 and SBDS is mediated by an intrinsically disordered insertion domain \\
\hline Author(s) & A sano, Nozomi; A tsuumi, Haruka; Nakamura, A kiyoshi; Tanaka, Y oshikazu; T anaka, I sao; Y ao, Min \\
\hline Citation & $\begin{array}{l}\text { Biochemical and Biophysical Research Communications, 443(4), 1251-1256 } \\
\text { https://doi.org/10.1016 } / \text {.bbrc.2013.12.143 }\end{array}$ \\
\hline Issue Date & $2014-01-24$ \\
\hline Doc URL & http://hdl.handle.net/2115/55598 \\
\hline Tyре & article (author version) \\
\hline File Information & EFL1_SBDS_journal.pdf \\
\hline
\end{tabular}

Instructions for use 


\title{
Direct interaction between EFL1 and SBDS is mediated by an intrinsically disordered insertion domain
}

\author{
Nozomi Asano ${ }^{1}$, Haruka Atsuumi $^{1}$, Akiyoshi Nakamura ${ }^{2}$, Yoshikazu Tanaka $^{2}$, Isao Tanaka ${ }^{2}$, \\ and Min $\mathrm{Yao}^{2} \boldsymbol{q}$
}

1 Graduate School of Life Sciences, Hokkaido University, Sapporo 060-0810

2 Faculty of Advanced Life Science, Hokkaido University, Sapporo 060-0810

I To whom correspondence should be addressed. Tel: +81-11-706-4481, Fax: +81-11-7064905, E-mail: yao@castor.sci.hokudai.ac.jp

Key words: Ribosome biogenesis, interaction, intrinsically disordered protein, isothermal titration calorimetry, circular dichroism

\section{HIGHLIGHTS}

- The interaction between EFL1 and SBDS was elucidated.

- EFL1 binds directly to SBDS with an association constant of $1.27 \times 10^{7} \mathrm{M}^{-1}$.

- The interaction is governed by the insertion domain of EFL1 and domain II - III of SBDS.

- The insertion domain of EFL1 acts as an intrinsically disordered domain.

- Roles of each domain of EFL1 and SBDS in Tif6 release are proposed. 


\begin{abstract}
Removal of anti-association factor, Tif6 (eIF6), by elongation factor-like 1 (EFL1) and Shwachman-Bodian-Diamond syndrome (SBDS) protein is a critical step in the late stage of ribosome maturation. Although EFL1 is known to have GTPase activity that is stimulated by SBDS, how they cooperatively trigger dissociation of Tif6 from the ribosome remains to be elucidated. In the present study, the interaction between EFL1 and SBDS was analyzed by size exclusion chromatography, gel shift assay, and isothermal titration calorimetry (ITC). The results showed that EFL1 interacted directly with SBDS. ITC experiments using domaintruncated mutants showed that the interaction between EFL1 and SBDS is governed by the insertion domain of EFL1 and domains II - III of SBDS. Circular dichroism spectroscopy showed that the insertion domain of EFL1 has a random structure in the absence of SBDS, whereas the disadvantageous entropy change observed on ITC suggested a fixed conformation coupled with complex formation with SBDS. Based on these observations together with those reported previously, we propose roles of EFL1 and SBDS in ribosomal maturation.
\end{abstract}




\section{INTRODUCTION}

Shwachman-Diamond syndrome is an autosomal recessive disorder characterized by hematological dysfunction, pancreatic exocrine insufficiency, skeletal abnormalities, and short stature [1]. Approximately $90 \%$ of Shwachman-Diamond syndrome cases are caused by mutations in the Shwachman-Bodian-Diamond syndrome $(S B D S)$ gene [2], which encodes a protein of approximately 250 amino acid residues. Orthologs of SBDS have been found in archaea, plants, and other eukaryotes. High-throughput affinity-capture mass spectrometry experiments identified potential interactions between SBDS and ribosome biogenesis factors [3], one of which was GTPase elongation factor-like 1 (EFL1). Genetic studies in Saccharomyces cerevisiae indicated that SBDS and EFL1 function cooperatively in a pathway to release the essential nucleolar factor, Tif6, from the late cytoplasmic pre-60S ribosomal subunit [4]. The removal of Tif6 - the yeast homolog of mammalian eukaryotic translation initiation factor 6 (eIF6) - is critical for late cytoplasmic maturation of the $60 \mathrm{~S}$ ribosomal subunit [5]. Tif6 acts as a ribosomal anti-association factor, which binds to the pre$60 \mathrm{~S}$ subunit to inhibit subunit joining by steric hindrance $[6,7,8]$. Therefore, dissociation of Tif6 from pre-60S ribosome is essential for enabling assembly into the $80 \mathrm{~S}$ subunit. Biochemical analysis showed that 60S-ribosome dependent GTP hydrolysis of EFL1 was stimulated by SBDS, and SBDS and EFL1 directly catalyzed Tif6 removal by a mechanism that required hydrolysis of GTP by EFL1 [9]. However, it remains to be elucidated how EFL1 and SBDS cooperatively trigger dissociation of Tif6 from the ribosome.

SBDS is composed of three domains (Fig. S1), and missense mutations of SBDS associated with Shwachman-Diamond syndrome were identified in all three domains [8]. Nuclear magnetic resonance (NMR) spectroscopy identified domain I of SBDS as an RNA binding site [10]. Moreover, it was reported that two mutants in domain II (R126T and K151N in 
human) were defective in triggering Tif6 release [9]. However, the roles of domains II and III were unclear. On the other hand, EFL1 shares $26.8 \%$ sequence identity with translation elongation factor 2 (EF2), and these two proteins share a ribosome binding site [11]. EFL1 triggers release of Tif6 from the pre-60S ribosome, whereas EF2 assists in the translocation of tRNA and mRNA from the A-site to the P-site of the ribosome. There is a marked difference in domain composition between these proteins, i.e., insertion of an extra $\sim 150$-residue domain in EFL1. ELF1 and EF2 are commonly composed of domains G, G', and II - V, but only EFL1 has the insertion region within domain II (Fig. S2) [12]. Although the insertion domain is expected to be an important determinant of the function, the details are still unclear.

Here, we performed interaction analysis of EFL1 and SBDS. The results showed that EFL1 binds directly with SBDS, in which the insertion region of EFL1 and domain II - III of SBDS dominate the interaction. The results of spectroscopic analysis taken together with the thermodynamic properties suggested conformational changes in the insertion region of EFL1 coupled with the interaction. Based on these observations, we discuss the significance of the interaction between EFL1 and SBDS for Tif6 removal. 


\section{MATERIALS AND METHODS}

\section{Plasmid construction}

The gene encoding EFL1 was amplified by PCR from $S$. cerevisiae genomic DNA using the primers EFL1-S-1 and EFL1-AS-1 (Supplementary Table S1), and inserted into the Sbfl-AscI sites of a modified pET26b vector (pECO-H2). In the resultant plasmid, a His-tag was attached to the N-terminus of the EFL1 gene. The plasmid for EFL1- $\triangle$ Ins (deletion mutant of the insertion region encoding residues 418 - 577) was constructed by the inverse PCR method using the EFL1 expression vector as the template and primers EFL1-S-2 and EFL1-AS-2. The DNA fragment for the insertion domain of EFL1 (EFL1-Ins; encoding residues 419 - 577) was amplified using the primers EFL1-S3 and EFL1-AS-3, followed by insertion into the Sbfl-AscI sites of a modified pET26b vector (pECO-GH1). In the resultant plasmid, a GSTtag followed by a TEV protease site and a His-tag were fused at the N-terminus and Cterminus, respectively.

The gene encoding SBDS was amplified by PCR from S. cerevisiae genomic DNA using the primers SBDS-S-4 and SBDS-AS-4, and inserted into the NdeI-XhoI sites of a modified pET28b vector (pDBHT-2), in which a His-tag was fused at the N-terminus. The coding sequences of SBDS domain I (encoding residues 1 - 94), domain II (encoding residues 95 172), domain III (encoding residues 173 - 250), domain I - II (encoding residues 1 - 172), and domain II - III (encoding residues 95 - 250) were amplified separately by PCR with the expression vector of SBDS as the template and the primers shown in Supplementary Table S1 and S2. The amplified DNA fragments encoding domains I, II, and I - II were inserted into the NdeI-XhoI sites of a modified pET28b vector (pET28M), in which His-tag was fused at the N-terminus. The DNA fragments encoding domain III and II - III were inserted into the NdeI-XhoI sites of the pET26b vector, in which His-tag was attached to the C-terminus. 


\section{Protein expression and purification}

Escherichia coli strain B834 (DE3) harboring the expression vector and pRARE2 was grown at $37^{\circ} \mathrm{C}$ in $\mathrm{LB}$ medium supplemented with $25 \mu \mathrm{g} / \mathrm{mL}$ kanamycin and $34 \mu \mathrm{g} / \mathrm{mL}$ chloramphenicol until the $\mathrm{OD}_{600}$ reached 0.6. To induce expression of the desired protein, IPTG was added at a final concentration of $0.25 \mathrm{mM}$. After incubation at $25^{\circ} \mathrm{C}$ for a further $18 \mathrm{~h}$ (exceptionally, for the expression of EFL1, $15^{\circ} \mathrm{C}$ for $24 \mathrm{~h}$ ), cells were harvested by centrifugation at $4500 \times \mathrm{g}$ for $10 \mathrm{~min}$ at $4^{\circ} \mathrm{C}$. Cells expressing EFL1 and the mutants were resuspended in $50 \mathrm{mM}$ Tris- $\mathrm{HCl}(\mathrm{pH} 7.5), 300 \mathrm{mM} \mathrm{NaCl}, 1 \mathrm{mM} \mathrm{MgCl} 2,10 \%$ (v/v) glycerol, $1 \mathrm{mg} / \mathrm{mL}$ lysozyme, and $0.1 \mathrm{mg} / \mathrm{mL}$ DNase I. Cells expressing SBDS and the mutants were resuspended in $50 \mathrm{mM}$ Tris- $\mathrm{HCl}(\mathrm{pH} 7.5), 300 \mathrm{mM} \mathrm{NaCl}, 10 \%(\mathrm{v} / \mathrm{v})$ glycerol, $1 \mathrm{mg} / \mathrm{mL}$ lysozyme, $0.1 \mathrm{mg} / \mathrm{mL}$ DNase I, and $0.1 \mathrm{mg} / \mathrm{mL}$ RNase A. Resuspended cells were disrupted by sonication, followed by centrifugation at $40000 \times g$ for $1 \mathrm{~h}$ at $10^{\circ} \mathrm{C}$.

EFL1 and EFL1- $\triangle$ Ins were purified on a HisTrap HP column (GE Healthcare) and HiLoad 16/60 Superdex 200-pg column (GE Healthcare). EFL1-Ins was purified on a HisTrap HP column (GE Healthcare), followed by removal of the GST-tag by digestion with TEV protease. EFL1-Ins without the GST-tag was further purified on a HisTrap HP column and HiLoad 16/60 Superdex 200-pg column.

SBDS and its truncated mutants were purified on a HisTrap HP column and HiLoad 26/60 Superdex 75-pg column. Exceptionally, SBDS-domain I was purified by three steps using a HisTrap HP column, HiTrap Heparin HP column, and HiLoad 26/60 Superdex 75-pg column.

\section{Gel filtration analyses}

Aliquots of $150 \mu \mathrm{L}$ consisting of $2.5 \mathrm{nmol}$ EFL1 and $8.5 \mathrm{nmol}$ SBDS were loaded onto a 
HiLoad 10/300 Superdex 200-pg column (GE Healthcare) pre-equilibrated with $20 \mathrm{mM}$ Tris$\mathrm{HCl}(\mathrm{pH} 7.5), 150 \mathrm{mM} \mathrm{NaCl}, 1 \mathrm{mM} \mathrm{MgCl}_{2}, 5 \%$ (v/v) glycerol, and $5 \mathrm{mM} \beta$-mercaptoethanol. Control experiments using each protein were also performed under the same conditions. Peak fractions were analyzed by SDS-PAGE, followed by staining with Coomassie brilliant blue R-250.

\section{Gel shift assay}

Gel shift assay was performed in $5-\mu \mathrm{L}$ reaction mixtures containing $20 \mathrm{mM}$ Tris- $\mathrm{HCl}(\mathrm{pH} 7.5)$, $150 \mathrm{mM} \mathrm{NaCl}, 1 \mathrm{mM} \mathrm{MgCl} 2,5 \%$ (v/v) glycerol, $5 \mathrm{mM} \beta$-mercaptoethanol, and the desired amounts of EFL1 and SBDS. Reaction mixtures were loaded onto a 3\% - 10\% native gradient polyacrylamide gel (PAGEL NPG-310L; ATTO). Electrophoresis conditions were as follows: temperature, $4^{\circ} \mathrm{C}$; power voltage, $100 \mathrm{~V}$; and electrophoresis buffer, $50 \mathrm{mM}$ Tris-MES (pH 8.0) and $10 \mathrm{mM} \mathrm{Mg}(\mathrm{OAc})_{2}$. Proteins were visualized using SYPRO® Ruby Protein gel stain.

\section{Isothermal titration calorimetry}

All isothermal titration calorimetry (ITC) measurements were carried out with a VP-ITC System (MicroCal). Proteins were dialyzed against a buffer containing $20 \mathrm{mM}$ Tris-HCl (pH 7.5), $150 \mathrm{mM} \mathrm{NaCl}, 1 \mathrm{mM} \mathrm{MgCl} 2,10 \%$ (v/v) glycerol, and $5 \mathrm{mM} \beta$-mercaptoethanol at $4^{\circ} \mathrm{C}$. All measurements were conducted at $30^{\circ} \mathrm{C}$ and protein solutions were degassed under vacuum prior to use. The cell was filled with $\sim 5 \mu \mathrm{M}$ full-length EFL1, EFL1- $\Delta$ Ins or $\sim 2.5 \mu \mathrm{M}$ EFL1Ins, and a syringe was filled with $\sim 50 \mu \mathrm{M}$ full-length SBDS or each truncated SBDS. The solution of SBDS was injected 25 times in portions of $10 \mu \mathrm{L}$ over $20 \mathrm{~s}$. The data were analyzed using the program ORIGIN (MicroCal). 


\section{Circular dichroism measurements}

Circular dichroism (CD) spectra were measured on a JASCO J-720 spectropolarimeter (JASCO) in a quartz cell with an optical path length of $2 \mathrm{~mm}$. The CD spectra were obtained by taking the average of four scans made from 300 to $190 \mathrm{~nm}$ and normalized to molar ellipticities by protein concentrations.

\section{Model building of EFL1-Tif6-bound ribosome}

The binding position of Tif6 on EF2-60S complex was obtained by superposing 60S ribosome subunits of eIF6(Tif6)-60S (PDB code: 4A18 [13]) and EF2-60S complex structure (PDB code: 1S1H and 1S1I [14]) using the program PyMoL (The PyMOL Molecular Graphics System, Schrödinger, LLC, New York, NY). The final model of the EFL1-Tif6-bound 60S ribosome subunit was built by superposing the crystal structure of Tif6 (PDB code: 1G62 [6]) onto the Tif6-EF2-60S model [15]. 


\section{RESULTS}

\section{Interaction between EFL1 and SBDS}

To investigate the details of the interaction between EFL1 and SBDS, the binding of EFL1 with SBDS was analyzed by size exclusion chromatography (SEC), gel shift assay, and isothermal titration calorimetry (ITC). Figure 1A shows the results of SEC. Compared with EFL1 and SBDS eluted with peaks at $11.19 \mathrm{~mL}$ and $15.15 \mathrm{~mL}$, respectively, the mixture showed a slightly earlier peak at $11.11 \mathrm{~mL}$. SDS-PAGE showed that both EFL1 and SBDS were contained in the peak of mixed sample (Fig. 1B), indicating the direct interaction between EFL1 and SBDS. Gel shift assay also clearly showed the interaction between EFL1 and SBDS (Fig. 1C). With increasing concentration of SBDS, the migration speed of EFL1 was significantly slowed. As EFL1 has GTPase activity, these experiments were also performed in the presence of GDPNP (an analog of GTP). However, there were no significant differences from the results in the absence of GTP (Fig. 1A). These results indicated that EFL1 and SBDS directly interact with each other in a GTP-independent manner. Next, we determined the thermodynamic parameters of the interaction by ITC (Fig. 1C and Table 1). The association constant and stoichiometry were $12.7 \times 10^{6} \mathrm{M}^{-1}$ and 0.94 , respectively, indicating that EFL1 binds to SBDS with a molar ratio of 1:1. The binding enthalpy, entropy, and Gibbs's energy were calculated to be $-14.0 \mathrm{kcal} \mathrm{mol}^{-1},-13.5 \mathrm{cal} \mathrm{mol}^{-1} \mathrm{~K}^{-1}$, and $-9.86 \mathrm{kcal}$ $\mathrm{mol}^{-1}$, respectively, indicating that the interaction between EFL1 and SBDS is driven by enthalpy and is entropically disadvantageous.

\section{Identification of the binding region in EFL1}

EFL1 shares sequence similarity with translation elongation factors, EF-G and EF-2 (Fig. 2A and Supplementary Fig. S2). These proteins commonly consist of the G domain (domain I), 
which binds with and hydrolyzes GTP, the G' domain, and domains II to V (Fig. S2). On the other hand, EFL1 has a characteristic insertion domain of 160 amino acids within domain II. To clarify the contribution of the insertion domain to the interaction with SBDS, the insertion domain of EFL1 (EFL1-Ins; Gly ${ }^{419}-\mathrm{Glu}^{577}$ ) and a mutant protein in which the insertion domain was deleted (EFL1- $\Delta$ Ins) were prepared, and their affinities with SBDS were evaluated by ITC (Fig. 2B). EFL1-Ins bound with SBDS, whereas no significant interaction was observed for EFL1- $\Delta$ Ins. The association constant, $11.8 \times 10^{6} \mathrm{M}^{-1}$ of EFL1-Ins, did not decrease markedly compared with that of EFL1 (Table 2). The thermodynamic parameters were binding enthalpy of $-16.7 \mathrm{kcal} \mathrm{mol}^{-1}$ and binding entropy of $-22.8 \mathrm{cal} \mathrm{mol}^{-1} \mathrm{~K}^{-1}$, indicating that EFL1-Ins binds to SBDS in an enthalpy-driven manner, which was the same as that of EFL1. Furthermore, EFL1 and EFL1 $\triangle$ Ins showed similar circular dichroism (CD) spectra, suggesting that truncation of the insertion domain did not affect the folding of EFL1

(Fig. 3). These observations indicated that the insertion region of EFL1 plays a pivotal role in the interaction with SBDS. The CD spectrum of EFL1-Ins showed a weak negative peak around $220 \mathrm{~nm}$ and strong negative $\mathrm{CD}$ around $200 \mathrm{~nm}$ (Fig. 3). These spectral features indicated that the insertion domain has a random structure with a small quantity of $\beta$-strands.

\section{Identification of the binding region in $S B D S$}

It has been reported that SBDS is composed of three domains with weak contacts among them $[8,9,10]$. As SBDS family proteins share a similar structure and sequence, ScSBDS is expected to consist of domain I (Met1 - Gln94), domain II (Leu95 - Ala172), and domain III (Lys173 - Asn250) (Fig. S1). Therefore, we prepared five domain-truncated mutants of SBDS (SBDS I, SBDS II, SBDS III, SBDS I - II, and SBDS II - III), and their interactions with EFL1 were evaluated by ITC (Fig. 4 and Table 3). 
SBDS II - III showed significant interaction with EFL1, whereas domain I - II did not (Fig. 4A and B). The association constant of SBDS II - III with EFL1 was determined to $1.19 \times 10^{6}$ $\mathrm{M}^{-1}$. The thermodynamic parameters indicated an enthalpy-driven and entropically disadvantageous interaction as observed in the interaction between intact SBDS and EFL1. Despite the observations in SBDS II-III, none of the other SBDS mutants showed significant interactions with EFL1 by themselves. These results indicated that both domains II and III of SBDS contribute to the interaction with EFL1. Taken together, these results indicated that the insertion domain of EFL1 and both domains II and III of SBDS dominate the enthalpy-driven entropically disadvantageous interaction between EFL1 and SBDS.

\section{Discussion}

Biological implications of SBDS-EFL1 interaction

Both SBDS and EFL1 were bound with the ribosome by itself [9]. It has been reported that EFL1 binds at the same site the on ribosome as EF2 [11], whereas the site for SBDS binding has not yet been identified. Our results showed that EFL1 interacted with SBDS directly, and this is the first study demonstrating the detailed interaction between them. The direct interaction indicated that SBDS binds in the vicinity of the EF2/EFL1 binding site on the ribosome. Oliveira et al. demonstrated that SBDS interacted with ribosomal RNA via domain I [10]. In addition, Finch et al. showed that the motion of domain I of SBDS was independent of domain II - III [9]. Our results demonstrated a significant role of domain II - III of SBDS in the interaction with EFL1. Taken together, these results suggest that domains I and II - III of SBDS are likely to act as functionally independent domains; i.e., domain I and domain II III bind to the ribosome and EFL1, respectively. EFL1 competes with EF2 for binding at the ribosomal GTPase binding site. This site will be occupied by EFL1 during ribosome 
biogenesis, whereas EF2 binds there in the elongation step during translation. The interaction between SBDS and EFL1 may facilitate predominant recruitment of EFL1 in the final maturation process of ribosome biogenesis.

To discuss the significance of the SBDS - EFL1 interaction in release of Tif6, a structural model of Tif6-EFL1-60S complex was constructed from the crystal structures of Tif6-60S complex and EF2-60S complex (Supplementary Fig. S3). In the model, EFL1 is positioned adjacent to Tif6, in which domain II of EFL1 is expected to show extensive interactions with Tif6. The insertion domain of EFL1, which is located within domain II, is necessary for the interaction with SBDS. Therefore, the insertion domain is likely to be toward Tif6. As the insertion domain is recognized by SBDS, the binding site of SBDS on the ribosome is expected to be close to the Tif6 binding site. Taken together, it is plausible that both EFL1 and SBDS are positioned in close proximity to Tif6 on the ribosome. However, the results of size exclusion chromatography indicated that Tif6 interacts with neither EFL1 nor SBDS directly (Supplementary Fig. S4). Biochemical analyses demonstrated that the presence of both EFL1 and SBDS, and GTP hydrolysis of EFL1 are all essential for the release of Tif6. Moreover, the presence of SBDS enhances the GTPase activity of EFL1 [9,16]. SBDS may increase the GTPase activity of EFL1 through complex formation. This is supported by the report that substitution in the domain II - III of SBDS resulted in a significant decrease in GTPase activity of EFL1 [9]. The GTPase activity would induce a conformational change of EFL1 as observed from other translational factors possessing GTPase activity, e.g., EF2 [12,17]. As Tif6 did not interact with EFL1 directly (Supplementary Fig. S3), the conformational change of EFL1 is likely to trigger release of Tif6 indirectly. As reported for EF2, the conformational change of EFL1 may induce rearrangement of the ribosome, which may result in the release of Tif6. These are consistent with the mechanism proposed 
previously [4].

\section{Role of the insertion domain of EFL1}

Although EFL1 and EF2 bind to the identical site on the ribosome, they have different functions [11]. They are clearly distinguished each other by the presence or absence of an insertion sequence within domain II. The CD spectra showed that the insertion domain of EFL1 (EFL1-Ins) was a random structure. On the other hand, ITC experiments showed that the interaction between EFL1 and SBDS was entropically disadvantageous. These observations indicate that the random structure of the insertion domain of EFL1 became static upon SBDS binding. It is likely that the insertion domain acts as an intrinsically disordered protein. Similar structural transition from a flexible to a rigid form coupled with ligand binding was reported previously for initiation factors [18]. The GTPase activity of EFL1 necessary for Tif6 release is enhanced by the presence of SBDS [9]. Our results indicated a direct interaction of the insertion domain of EFL1 with SBDS accompanying conformational transition. These observations suggest that the fixed conformation is important for the release of Tif6. EFL1 may be necessary to acquire the intrinsically disordered domain as an insertion domain to express these two different functions, i.e., inhibition of EF2-like activity utilizing the unfolded structure and promoting Tif6 release in the fixed form. 


\section{References}

[1] G.W. Hall, P. Dale, J.A. Dodge, Shwachman-Diamond syndrome: UK perspective, Arch Dis Child 91 (2006) 521-524.

[2] G. Horiguchi, H. Kodama, K. Iba, Mutations in a gene for plastid ribosomal protein S6like protein reveal a novel developmental process required for the correct organization of lateral root meristem in Arabidopsis, Plant J 33 (2003) 521-529.

[3] N.J. Krogan, G. Cagney, H. Yu, G. Zhong, X. Guo, A. Ignatchenko, J. Li, S. Pu, N. Datta, A.P. Tikuisis, T. Punna, J.M. Peregrin-Alvarez, M. Shales, X. Zhang, M. Davey, M.D. Robinson, A. Paccanaro, J.E. Bray, A. Sheung, B. Beattie, D.P. Richards, V. Canadien, A. Lalev, F. Mena, P. Wong, A. Starostine, M.M. Canete, J. Vlasblom, S. Wu, C. Orsi, S.R. Collins, S. Chandran, R. Haw, J.J. Rilstone, K. Gandi, N.J. Thompson, G. Musso, P. St Onge, S. Ghanny, M.H. Lam, G. Butland, A.M. Altaf-Ul, S. Kanaya, A. Shilatifard, E. O'Shea, J.S. Weissman, C.J. Ingles, T.R. Hughes, J. Parkinson, M. Gerstein, S.J. Wodak, A. Emili, J.F. Greenblatt, Global landscape of protein complexes in the yeast Saccharomyces cerevisiae, Nature 440 (2006) 637-643.

[4] T.F. Menne, B. Goyenechea, N. Sanchez-Puig, C.C. Wong, L.M. Tonkin, P.J. Ancliff, R.L. Brost, M. Costanzo, C. Boone, A.J. Warren, The Shwachman-Bodian-Diamond syndrome protein mediates translational activation of ribosomes in yeast, Nat Genet 39 (2007) 486-495.

[5] U. Basu, K. Si, J.R. Warner, U. Maitra, The Saccharomyces cerevisiae TIF6 gene encoding translation initiation factor 6 is required for $60 \mathrm{~S}$ ribosomal subunit biogenesis, Mol Cell Biol 21 (2001) 1453-1462.

[6] C.M. Groft, R. Beckmann, A. Sali, S.K. Burley, Crystal structures of ribosome antiassociation factor IF6, Nat Struct Biol 7 (2000) 1156-1164. 
[7] M. Ceci, C. Gaviraghi, C. Gorrini, L.A. Sala, N. Offenhauser, P.C. Marchisio, S. Biffo, Release of eIF6 (p27BBP) from the 60S subunit allows 80 S ribosome assembly, Nature 426 (2003) 579-584.

[8] C. Shammas, T.F. Menne, C. Hilcenko, S.R. Michell, B. Goyenechea, G.R. Boocock, P.R. Durie, J.M. Rommens, A.J. Warren, Structural and mutational analysis of the SBDS protein family. Insight into the leukemia-associated Shwachman-Diamond Syndrome, J Biol Chem 280 (2005) 19221-19229.

[9] A.J. Finch, C. Hilcenko, N. Basse, L.F. Drynan, B. Goyenechea, T.F. Menne, A. Gonzalez Fernandez, P. Simpson, C.S. D'Santos, M.J. Arends, J. Donadieu, C. BellanneChantelot, M. Costanzo, C. Boone, A.N. McKenzie, S.M. Freund, A.J. Warren, Uncoupling of GTP hydrolysis from eIF6 release on the ribosome causes ShwachmanDiamond syndrome, Genes Dev 25 (2011) 917-929.

[10] J.F. de Oliveira, M.L. Sforca, T.M. Blumenschein, M.B. Goldfeder, B.G. Guimaraes, C.C. Oliveira, N.I. Zanchin, A.C. Zeri, Structure, dynamics, and RNA interaction analysis of the human SBDS protein, J Mol Biol 396 (2010) 1053-1069.

[11] J.S. Graindorge, J.C. Rousselle, B. Senger, P. Lenormand, A. Namane, F. Lacroute, F. Fasiolo, Deletion of EFL1 results in heterogeneity of the 60 S GTPase-associated rRNA conformation, J Mol Biol 352 (2005) 355-369.

[12] R. Jorgensen, P.A. Ortiz, A. Carr-Schmid, P. Nissen, T.G. Kinzy, G.R. Andersen, Two crystal structures demonstrate large conformational changes in the eukaryotic ribosomal translocase, Nat Struct Biol 10 (2003) 379-385.

[13] S. Klinge, F. Voigts-Hoffmann, M. Leibundgut, S. Arpagaus, N. Ban, Crystal structure of the eukaryotic 60S ribosomal subunit in complex with initiation factor 6 , Science $334(2011) 941-948$. 
[14] C.M. Spahn, M.G. Gomez-Lorenzo, R.A. Grassucci, R. Jorgensen, G.R. Andersen, R. Beckmann, P.A. Penczek, J.P. Ballesta, J. Frank, Domain movements of elongation factor eEF2 and the eukaryotic $80 \mathrm{~S}$ ribosome facilitate tRNA translocation, EMBO J 23 (2004) 1008-1019.

[15] M. Gartmann, M. Blau, J.P. Armache, T. Mielke, M. Topf, R. Beckmann, Mechanism of eIF6-mediated inhibition of ribosomal subunit joining, J Biol Chem 285 (2010) $14848-14851$.

[16] A. Gijsbers, A. Garcia-Marquez, A. Luviano, N. Sanchez-Puig, Guanine nucleotide exchange in the ribosomal GTPase EFL1 is modulated by the protein mutated in the Shwachman-Diamond Syndrome, Biochem Biophys Res Commun 437 (2013) 349354.

[17] D.J. Taylor, J. Nilsson, A.R. Merrill, G.R. Andersen, P. Nissen, J. Frank, Structures of modified eEF2 80S ribosome complexes reveal the role of GTP hydrolysis in translocation, EMBO J 26 (2007) 2421-2431.

[18] Z. Gai, Y. Kitagawa, Y. Tanaka, N. Shimizu, K. Komoda, I. Tanaka, M. Yao, The binding mechanism of eIF2beta with its partner proteins, eIF5 and eIF2Bepsilon, Biochem Biophys Res Commun 423 (2012) 515-519. 


\section{Figure legends}

Fig. 1 Interaction between EFL1 and SBDS. (A) Size exclusion chromatography of EFL1 incubated with SBDS. Chromatograms of EFL1, SBDS, and their complex in the presence of GDPNP are also shown. The elution volumes are indicated at the tops of the peaks. (B) SDSPAGE of the peak fractions on size exclusion chromatography. Lane 1, EFL1; lane 2, SBDS; lane 3, EFL1-SBDS; lane 4, EFL1-SBDS in the presence of GDPNP. (C) Results of gel shift assay. A 50-pmol aliquot of EFL1 was incubated with different amounts of SBDS. Lane 1, EFL1 only; lanes 2-8, increasing amounts of $\operatorname{SBDS}(2,5,10,25,50,100,500,1000$ pmol). (D) Thermogram of interaction between EFL1 and SBDS.

Fig. 2 Interaction of EFL1 mutants for SBDS. (A) Schematic overview of the EFL1 variants. The insertion sequence within domain II is shown in light gray. (B) Thermograms of titration for SBDS of EFL1- $\triangle$ Ins (left) and EFL1-Ins (right).

Fig. 3 CD spectra of EFL1 variants. Gray line; EFL1 wild type, black line; EFL1- $\Delta$ Ins, dotted black line; EFL1-Ins.

Fig. 4 Interaction of SBDS mutants for EFL1. (A) Schematic overview of the SBDS variants. $(B-F)$ Thermograms of titration of SBDS variants for EFL1. B; SBDS I- II, C; SBDS II - III, D; SBDS I, E; SBDS II, F; SBDS III.

Fig. S1 Structure of Human SBDS. PDB code; 2L9N

Fig. S2 Amino acid sequence alignment of EFL1 and EF2. Domains are represented by 
color bars above. Secondary structure elements of S. cerevisiae EF2 are also shown.

Fig. S3 Structure model of ribosome-Tif6-EFL1/EF2 ternary complex. Red spheres represent the site in which the insertion domain is located. Tif6 and EF2 are colored as follows. blue, Tif6; light brown, domain IV of EF2/EFL1; yellow, domain III of EF2/EFL1; pink, V of EF2/EFL1; green, domain II of EF2/EFL1; purple, domain G(I) of EF2/EFL1; cyan, domain G' of EFL1/EF2; gray, ribosomal RNA.

Fig. S4 Size exclusion chromatography of Tif6 incubated with EFL1 and SBDS. Chromatograms of Tif6, EFL1, and SBDS are also shown. 
Table 1. Affinity and thermodynamic parameters of binding between SBDS and EFL1 at $30^{\circ} \mathrm{C}$

\begin{tabular}{cccccccc}
\hline EFL1 & SBDS & $\mathrm{N}$ & $\begin{array}{c}K_{\mathrm{b}} \\
\left(\times 10^{6} \mathrm{M}^{-1}\right)\end{array}$ & $\begin{array}{c}K_{\mathrm{d}}^{\mathrm{a}} \\
(\mathrm{nM})\end{array}$ & $\begin{array}{c}\Delta \mathrm{H} \\
\left(\mathrm{kcal} \cdot \mathrm{mol}^{-1}\right)\end{array}$ & $\begin{array}{c}\Delta \mathrm{S} \\
\left(\mathrm{cal} \cdot \mathrm{mol}^{-1} \cdot \mathrm{K}^{-1}\right)\end{array}$ & $\begin{array}{c}\Delta \mathrm{G}^{\mathrm{b}} \\
\left(\mathrm{kcal} \cdot \mathrm{mol}^{-1}\right)\end{array}$ \\
\hline \hline $\begin{array}{c}\text { full } \\
\text { length }\end{array}$ & $\begin{array}{c}\text { full } \\
\text { length }\end{array}$ & $\begin{array}{c}0.94 \pm \\
0.007\end{array}$ & $12.7 \pm 1.51$ & 78.7 & $-14.0 \pm 0.17$ & -13.5 & 9.86 \\
\hline
\end{tabular}

${ }^{\mathrm{a}} K_{\mathrm{d}}=1 / K_{\mathrm{b}}$

${ }^{\mathrm{b}} \Delta \mathrm{G}=-\mathrm{RT} \ln K_{\mathrm{b}}=\Delta \mathrm{H}-\mathrm{T} \Delta \mathrm{S}$

Table 2. Affinities and thermodynamic parameters of binding between SBDS and EFL1 mutants at $30^{\circ} \mathrm{C}$

\begin{tabular}{|c|c|c|c|c|c|c|c|}
\hline EFL1 & SBDS & $\mathrm{N}$ & $\begin{array}{c}K_{\mathrm{b}} \\
\left(\times 10^{6} \mathrm{M}^{-1}\right)\end{array}$ & $\begin{array}{c}K_{\mathrm{d}} \\
(\mathrm{nM})\end{array}$ & $\begin{array}{c}\Delta \mathrm{H} \\
\left(\mathrm{kcal} \cdot \mathrm{mol}^{-1}\right)\end{array}$ & $\begin{array}{c}\Delta \mathrm{S} \\
\left(\mathrm{cal} \cdot \mathrm{mol}^{-1} \cdot \mathrm{K}^{-}\right. \\
1)\end{array}$ & $\begin{array}{c}\Delta \mathrm{G} \\
\left(\mathrm{kcal} \cdot \mathrm{mol}^{-1}\right)\end{array}$ \\
\hline$\Delta \mathrm{Ins}$ & $\begin{array}{c}\text { full } \\
\text { length }\end{array}$ & $\mathrm{ND}^{\mathrm{a}}$ & ND & ND & ND & ND & ND \\
\hline Ins & $\begin{array}{l}\text { full } \\
\text { length }\end{array}$ & $\begin{array}{c}0.778 \pm \\
0.02\end{array}$ & $11.8 \pm 2.45$ & 84.7 & $-16.7 \pm 0.51$ & -22.8 & 9.81 \\
\hline
\end{tabular}

${ }^{\mathrm{a}} \mathrm{ND}$ : not detected.

Table 3. Affinities and thermodynamic parameters of binding between SBDS mutants and EFL1 at $30^{\circ} \mathrm{C}$

\begin{tabular}{|c|c|c|c|c|c|c|c|}
\hline EFL1 & SBDS & $\mathrm{N}$ & $\begin{array}{c}K_{\mathrm{b}} \\
\left(\times 10^{6} \mathrm{M}^{-1}\right)\end{array}$ & $\begin{array}{c}K_{\mathrm{d}} \\
(\mathrm{nM})\end{array}$ & $\begin{array}{c}\Delta \mathrm{H} \\
\left(\mathrm{kcal} \cdot \mathrm{mol}^{-1}\right)\end{array}$ & $\begin{array}{c}\Delta \mathrm{S} \\
\left(\mathrm{cal} \cdot \mathrm{mol}^{-1} \cdot \mathrm{K}^{-}\right. \\
1)\end{array}$ & $\begin{array}{c}\Delta \mathrm{G} \\
\left(\mathrm{kcal}^{\cdot} \mathrm{mol}^{-1}\right)\end{array}$ \\
\hline $\begin{array}{c}\text { full } \\
\text { length }\end{array}$ & I - II & ND & ND & ND & ND & ND & ND \\
\hline $\begin{array}{l}\text { full } \\
\text { length }\end{array}$ & II - III & $\begin{array}{c}0.803 \pm \\
0.03\end{array}$ & $1.19 \pm 1.69$ & 840 & $-10.6 \pm 0.52$ & -7.04 & 8.43 \\
\hline $\begin{array}{l}\text { full } \\
\text { length }\end{array}$ & I & ND & ND & ND & ND & ND & ND \\
\hline $\begin{array}{c}\text { full } \\
\text { length }\end{array}$ & II & ND & ND & ND & ND & ND & ND \\
\hline $\begin{array}{l}\text { full } \\
\text { length }\end{array}$ & III & ND & ND & ND & ND & ND & ND \\
\hline
\end{tabular}

${ }^{\mathrm{a}} \mathrm{ND}$ : not detected. 
Fig. 1

A

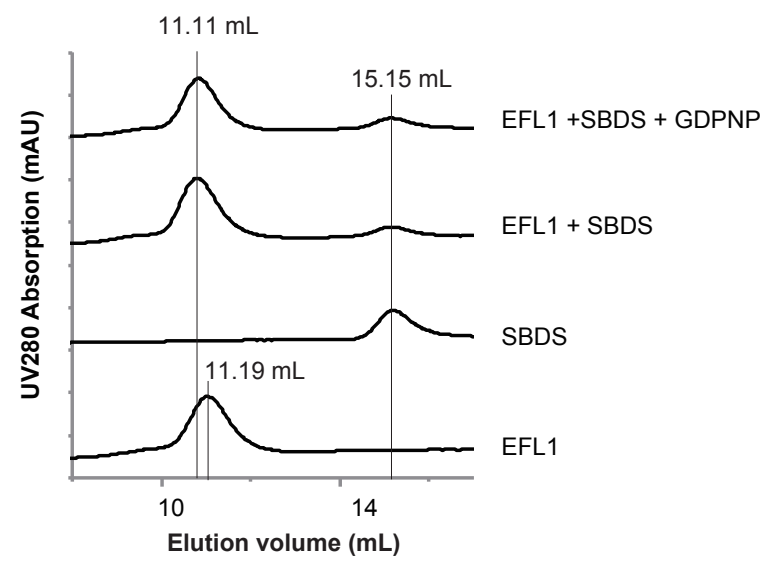

C

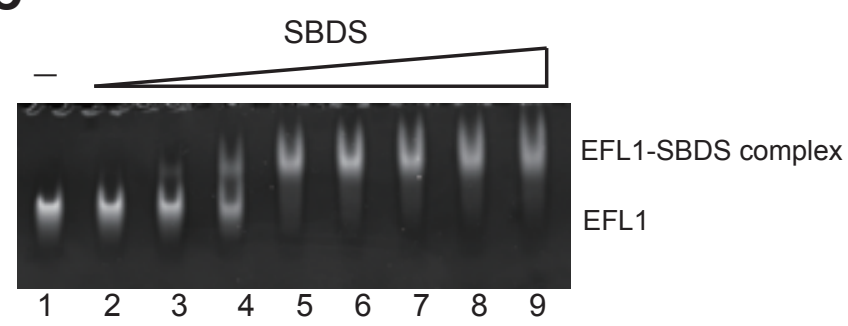

B

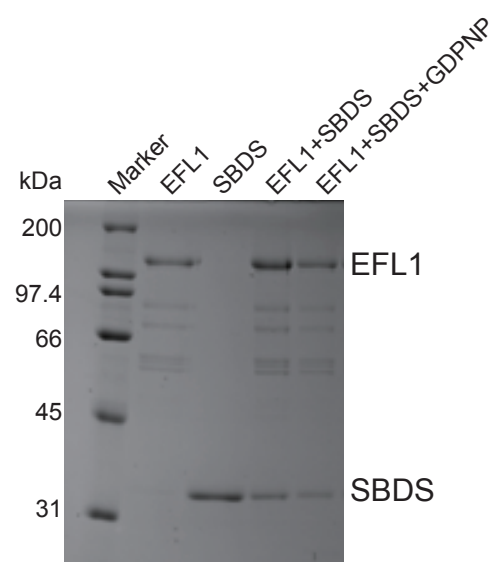

D

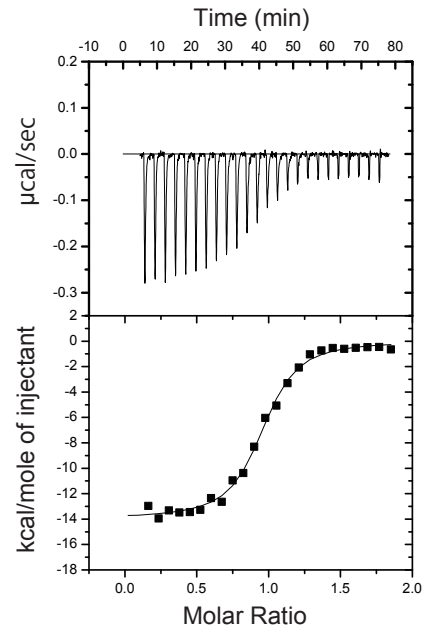

EFL1 vs SBDS 
Fig. 2

A

\begin{tabular}{|l|l|l|l|l|l|l|l|l|}
\hline EFL1 (I) & G' & II & Ins & II & III & IV & V & IV \\
\hline
\end{tabular}

EFL1- $\Delta$ Ins

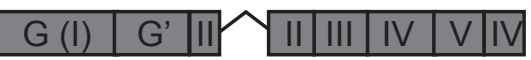

EFL1-Ins

Ins

B

EFL1- $\Delta$ Ins vs. SBDS

EFL1-Ins vs. SBDS

Time (min)

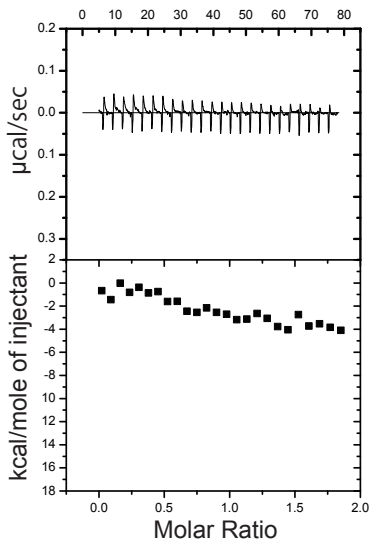

Time (min)

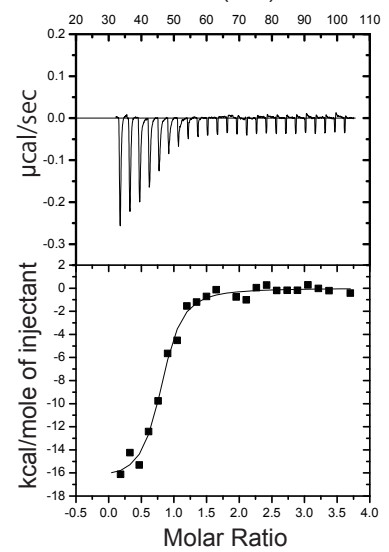


Fig. 3

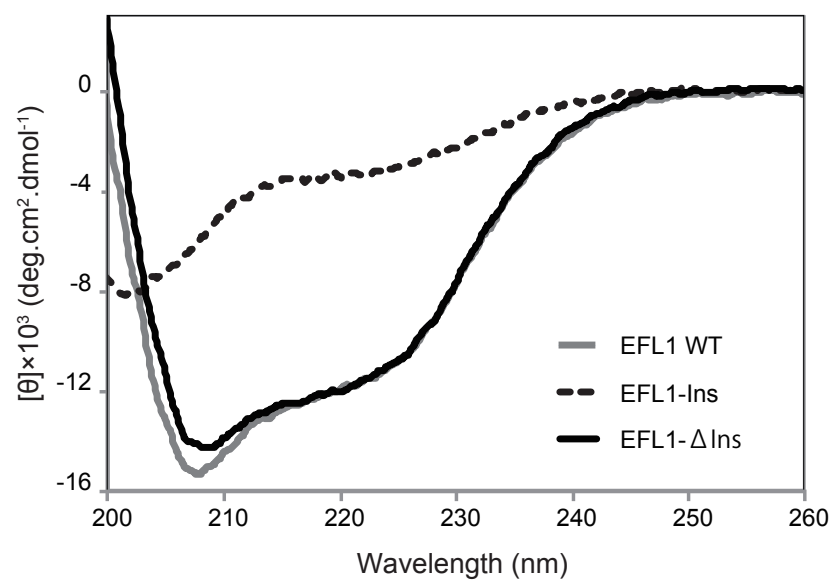




\section{Fig. 4}

A

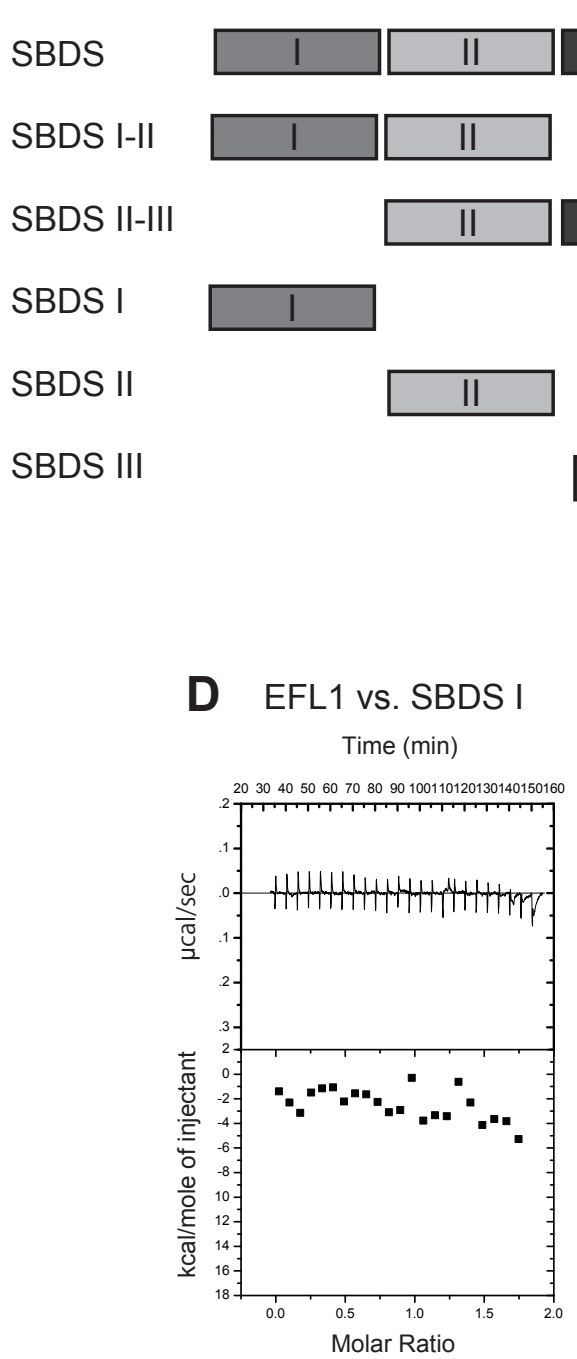

B EFL1 vs. SBDS I-II

Time (min)

III

III

III

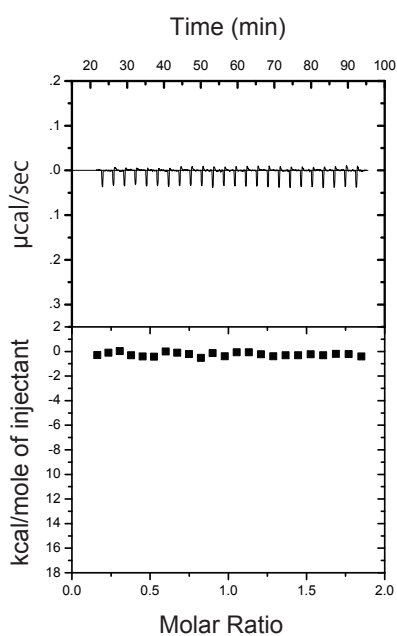

C EFL1 vs. SBDS II-III

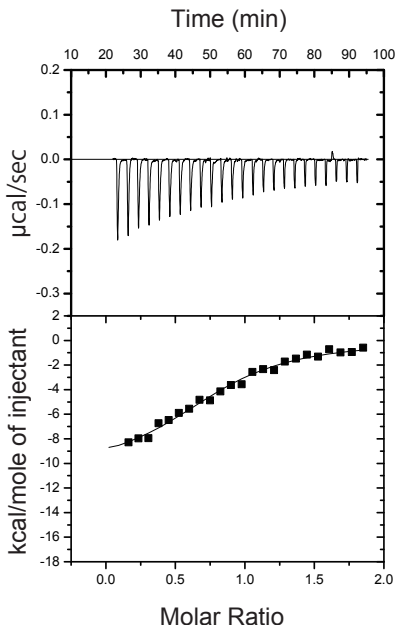

E EFL1 vs. SBDS ॥

Time (min)

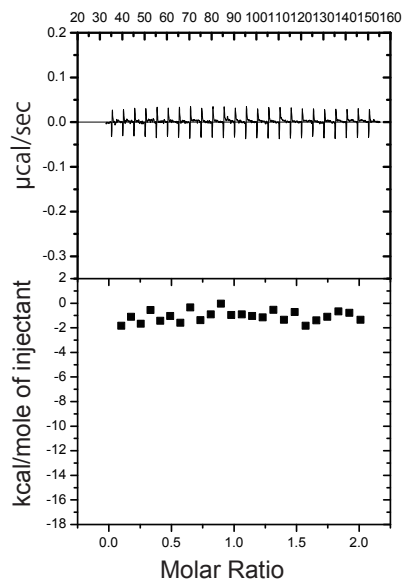

F EFL1 vs. SBDS III

Time (min)

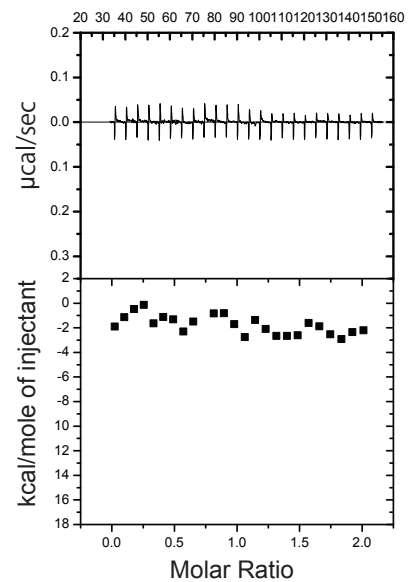




\section{Supplementary Fig. S1}

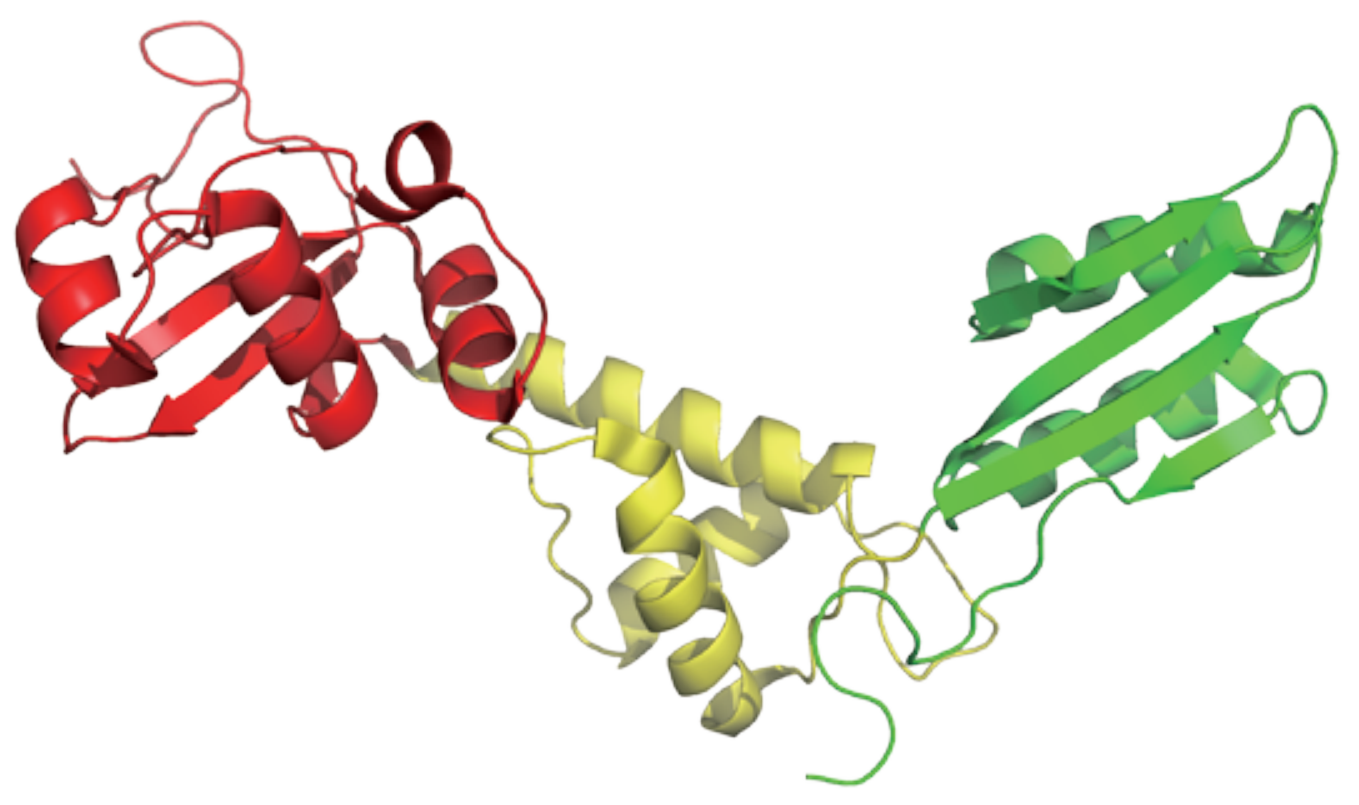

HsSBDS

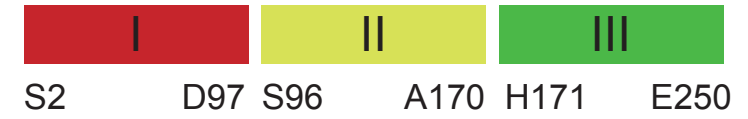




\section{Supplementary Fig. S2}






\section{Supplementary Fig. S3}

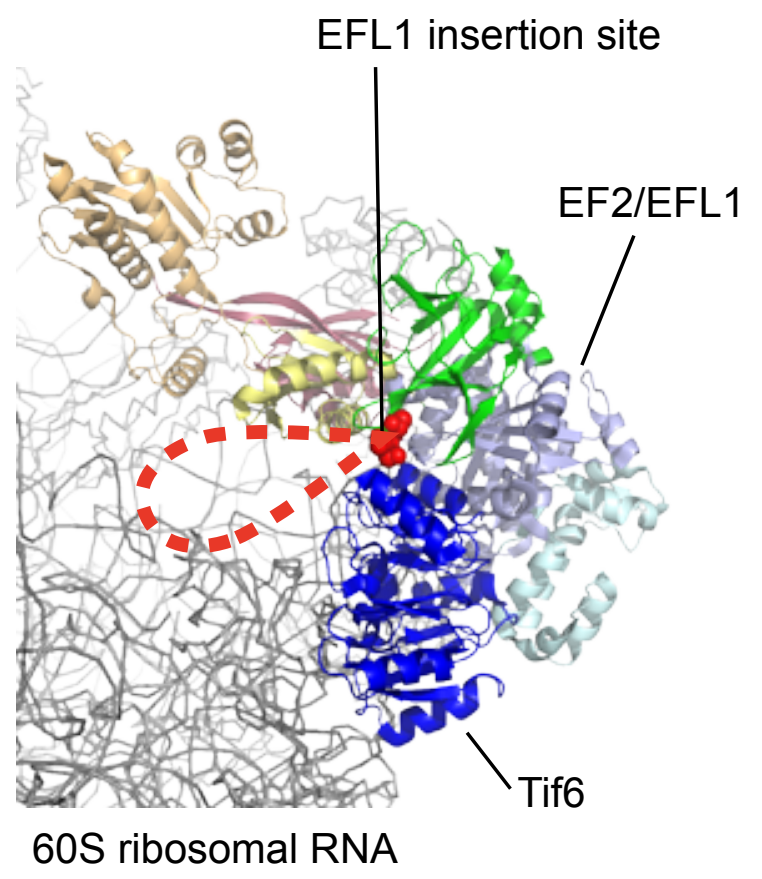




\section{Supplementary Fig. S4}

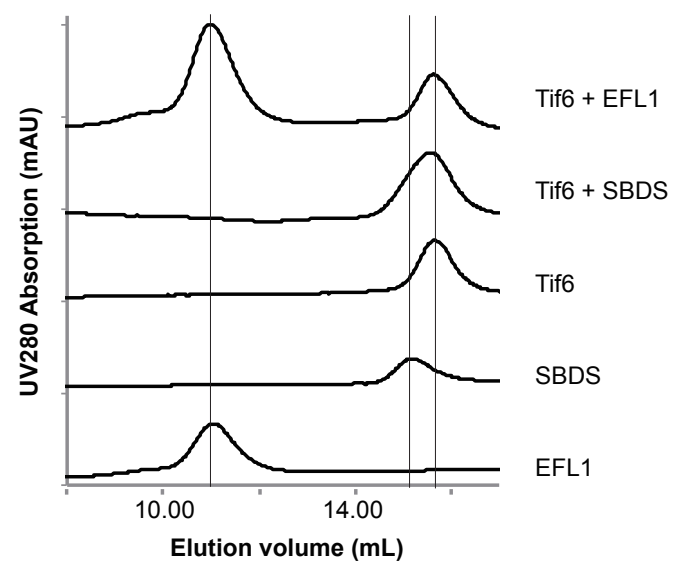


Supplementary Table S1. Primers used

\begin{tabular}{|l|l|}
\hline Name & Sequence \\
\hline S-1 & CCC ACC TGC AGG GTG GTA GCA TGC CTA GAG TGG AAT CG \\
\hline S-2 & CAA TTC CTA AGG AAG AAT GCT TGG TAG CAT \\
\hline S-3 & CCC ACC TGC AGG GTG GTA GCA TGG AAG AAT TAC CTG TTG AAT CTA AGA G \\
\hline S-4 & TGG TGC CGC GCG GCA GCC ATA TGC CTA TCA ATC AAC CGT \\
\hline S-5 & GGA ATT CCA TAT GCA ATT ATC GGA AAA AGA AAG ACA ATT AAT G \\
\hline S-6 & GGA ATT CCA TAT GGC GAA GAT GAA AGT CAA AGT G \\
\hline AS-1 & GGG TGG CGC GCC TTA ATT CTT TTT CAA AGT ACG TTG TTT TT \\
\hline AS-2 & TCT TCC TTA GGA ATT GAA AGC ATC TTA GAT \\
\hline AS-3 & GGG TGG CGC GCC TTC GTC AAA AAT ATC ATC TAC TTC ATC GTT C \\
\hline AS-4 & TGG TGG TGG TGG TGC TCG AGT TAG TTA TGC GTT GTA TTA TCT ATG AC \\
\hline AS-5 & CCG CTC GAG TTA TTG AAT CTC TCC CTT ATG CAT GA \\
\hline AS-6 & CCG CTC GAG TTA CGC CCT TAC TAT TGG AAT AAT TTG \\
\hline
\end{tabular}

Supplementary Table S2. Plasmids

\begin{tabular}{llll}
\hline Name & Vector & Construct & Primers \\
\hline EFL1 & pECO-H2 & His tag at N-terminus & S-1, AS-1 \\
EFL1 IIns & pECO-H2 & His tag at N-terminus & S-2, AS-2 \\
EFL1-Ins & pECO-GH1 & $\begin{array}{l}\text { GST tag - TEV S-3, AS-3 } \\
\text { protease site at } \\
\text { N-terminus, His tag at } \\
\text { C-terminus }\end{array}$ \\
& & His tag at N-terminus & S-4, AS-4 \\
SBDS & pDBHT-2 & His tag at N-terminus & S-4, AS-5 \\
SBDS domain I & pET28M & His tag at N-terminus & S-5, AS-6 \\
SBDS domain II & pET28M & His tag at C-terminus & S-6, AS-4 \\
SBDS domain III & pET26M & His tag at N-terminus & S-4, AS-6 \\
SBDS domain I - II & pET28M & His tag at C-terminus & S-5, AS-4 \\
\hline SBDS domain II - III & pET26M &
\end{tabular}

